\title{
EL SOLUTRENSE EN MADRID (ESPAÑA): ANÁLISIS BIBLIOGRÁFICO Y COMPARATIVO
}

\section{The Solutrean in Madrid (Spain): review and comparative literature}

\author{
Andrés A. Fernández Gómez ${ }^{1}$ y Anna Velasco Ortiz ${ }^{1}$
}

Recibido el 15 de marzo de 2013. Aceptado el 13 de septiembre de 2013

Resumen. En este trabajo ofrecemos una revisión crítica de todos los datos provenientes de las investigaciones paleolíticas en la Comunidad de Madrid (España) desde 1862, cuando Casiano de Prado descubrió las primeras herramientas líticas en asociación con huesos de animales. Evaluamos el origen de esos estudios en el centro de la Península Ibérica desde una visión historiográfica. Con un especial interés en los yacimientos solutrenses cerca de los ríos Manzanares y Jarama como El Sotillo, Valdocarros, Las Delicias, etc., y sus problemas, con el fin de proporcionar algunas conclusiones sobre este periodo en Madrid.

Palabras clave: Solutrense, Paleolítico superior, historia de la investigación, Comunidad de Madrid.

Abstract. In this paper we offer a critical review of all data coming from the Paleolithic researches in the Community of Madrid (Spain) since 1862, when Casiano de Prado discovered the first lithic tools in association with animals' bones. We evaluate the origin of those studies in the center of the Iberian Peninsula from a historiographical vision. With a specially interest at the Solutrean sites near the rivers Manzanares and Jarama such as EI Sotillo, Valdocarros, Las Delicias, etc., and their problems, in order to provide some conclusions about this period in Madrid.

Keywords: Solutrean, Upper Paleolithic, research history, Community of Madrid.

\section{INTRODUCCIÓN. LA INVESTIGACIÓN} DEL PALEOLÍTICO EN LA COMUNIDAD DE MADRID

Los primeros estudios paleolitistas de Madrid coinciden con el inicio de la Prehistoria en Europa (segunda mitad del s. XIX), siendo las terrazas del Manzanares, área de San Isidro del Campo, donde se produjeron dichos hallazgos (Santonja y Vega 2002; Wernert y Pérez de Barradas 1925). Destaca la labor de la Comisión del Mapa Geológico de España y los trabajos de Joaquín Ezquerra de Bayo y Mariano de la Paz Graells (1850) (Wernert y Pérez de Barradas 1925). Este último, registró y relacionó restos de elefantes (Tejar de las Ánimas) con proboscídeos aparecidos en la zona desde el s. XVIII (Puche Riart 2008; Wernert y Pérez de Barradas 1925: 3), pudiéndose considerar esta labor la primera excavación del Cuaternario en el Manzanares (Santonja et al. 2011).

Sin embargo, las primeras observaciones que establecieron una hipótesis respecto al ser humano se produjeron en el cerro de San Isidro, 1862, estableciéndose una relación entre la fauna extinta y la industria lítica asociada (Prado 1864; Santonja y Vega, 2002; Santonja et al. 2011). Ello se debió al trabajo estratigráfico desarrollado por Casiano de Prado a partir de los areneros de la terraza del río Manzanares en San Isidro (Prado 1864; Santonja y Vega, 2002; Santonja et al. 2000; 2011; Wernert y Pérez de Barradas 1925:33). 
Curiosamente, en 1850, Casiano de Prado ya había hallado fragmentos de sílex con rasgos morfológicos peculiares en los depósitos fluviales de San Isidro, que en sus propias palabras (Prado 1864:298) sólo entendió cuando volvió, en 1862, acompañado de Edouard de Verneuil y Louis Lartet. Se identificó al menos una pieza como obra indiscutible del ser humano (Santonja y Vega 2002; Santonja et al. 2000; Wernert y Pérez de Barradas 1925: 34).

Dichos hallazgos fueron rápidamente publicados (Casiano de Prado 1864; Verneuil y Lartet 1863) por ser los primeros de este tipo en el país $y$, a su vez, éste el tercer país donde se admitía la existencia de industria lítica en Europa (Puche Riart 2008; Santonja y Vega 2002; Santonja et al. 2000).

Por desgracia, el brillante inicio de la Prehistoria en Madrid no tuvo continuidad. Tras fallecer Casiano de Prado, los investigadores se interesaron más por la divulgación (Santonja et al. 2011: 31). Aunque la notoriedad de los hallazgos atrajo a investigadores, en muchos casos de otras nacionalidades, esto sólo produjo un goteo de artículos de escaso interés, pues la mayoría no aportaban nada nuevo. Destacó Juan Vilanova (1821-1893), discípulo de Casiano de Prado, quien intentó poner orden en las informaciones publicadas en la época (Puche Riart 2008) proponiendo, en 1975, una clasificación cultural para la Prehistoria peninsular, en la que no aparecía ningún resto solutrense (Ayarzagüena, 2002: 69).

Surgieron asociaciones interesadas en la Prehistoria como la Sociedad Antropológica Española (Ayarzagüena 2002: 62) y colecciones como las de José Quiroga o Emilio Rotondo (Maier 2000). Y se produjo la aparición del Solutrense como periodo cultural en España (Ayarzagüena 2002: 74) y la publicación de los hallazgos de Valdocarros (Villanova 1891) y San Isidro (Puche Riart 2008: 289).

La necesidad de establecer una metodología común a la hora de tratar los restos trajo la promulgación, en 1911, de la Ley de Excavaciones (Rasilla y Santamaría 2005), creándose la Comisión de Investigaciones Paleontológicas y Prehistóricas, CIPP (Rasilla y Santamaría 2005; Santonja y Vega 2002; Santonja et al. 2000). En ella estaban los cuaternaristas, geólogos y paleontólogos españoles más reconocidos, dando lugar al primer equipo multidisciplinar del país (RasiIla y Santamaría 2005).

Hugo Obermaier y Paul Wernert se unieron a ellos, en 1915, tras fijar su residencia en Madrid, debido al estallido de la I Guerra Mundial y a la imposibilidad de regresar a su actividad en Francia por su origen germánico (Santonja et al. 2011: 33).

Ellos retomaron los estudios en San Isidro (1911) e iniciaron la exploración sistemática del resto de areneros del Manzanares (Santonja y Vega 2002; Santonja et al. 2011; Wernert y Pérez de Barradas 1925). Pronto se unió a su labor, como becario del Museo Nacional de Ciencias Naturales, M.N.C.N., José Pérez de Barradas (1897-1980), colaborando en el descubrimiento de cuantiosos yacimientos. Asumiendo un papel más destacado, se dispuso a realizar la interpretación geológica del valle y la primera sintesis sobre la industria paleolítica (Santonja et al. 2011: 35).

El objetivo común era establecer el marco cronológico de las "civilizaciones" paleolíticas del valle e integrarlas en la secuencia histórico-cultural continental, diseñada por Obermaier (Santonja y Vega 2002; Santonja et al. 2000; 2011). En sus planteamientos consideraban la tipología como herramienta para definir los períodos culturales (Santonja y Vega 2002; Santonja et al. 2000; 2011), aunque sus escritos no fueron más allá del registro de las industrias y su asignación a un período (Santonja et al. 2011: 33). Este periodo duró hasta 1924 y los resultados tienen su expresión en la segunda edición de El Hombre Fósil (Obermaier 1925: 195-212).

Las discrepancias surgidas en el interior de la CIPP entre los geólogos-paleontólogos y prehistoriadores (Rasilla y Santamaría 2005; Santonja y Vega 2002; Santonja et al. 2010), por el distinto valor otorgado a las industrias líticas como fósil guía para identificar los periodos geológicos, afectaron al desarrollo de la Prehistoria madrileña (Santonja y Vega 2002; Santonja et al. 2010).

Así, Obermaier y Wernert orientaron su esfuerzo a otros proyectos arqueológicos y abandonaron, paulatinamente, las investigaciones referidas al Paleolítico madrileño (DíazAndreu 2000; Santonja y Vega 2002). Fue Pérez de Barradas, la figura más destacada de la Prehistoria madrileña, quien inició una variación en la orientación teórica de sus trabajos (Santonja y Vega 2002). Con el respaldo económico e institucional de la administración ocupó diversos cargos, siendo el último y más destacado el de director del Museo de Prehistoria de Madrid y el Servicio de Investigaciones Prehistóricas (Carrera y Martín Flores 2002).

Esta nueva fase, al principio, se caracterizó por la elaboración de interpretaciones generales basadas en los resultados anteriores y finalizó, como el mismo protagonista apunta, en 1931 (Pérez de Barradas, 1933-36: 3) debido a la importante reducción de las subvenciones y los recursos económicos del Servicio de Investigación Prehistórica (Santonja et al. 2011: 39).

La síntesis geológica diseñada por Pérez de Barradas tomó elementos de los estudios realizados por Hernández Pacheco y Royo, aunque sin admitir la edad miocena del denominado "cuaternario de arrastre lento" (Pérez de Barradas 1929: 161), y redefinió la secuencia de terrazas del Manzanares sin variar la cronología y clasificación de la industria (Santonja et al. 2011: 39).

En sus últimas actuaciones, ya en la segunda república, influenciado por Breuil, creó un nuevo sistema cultural acuñando el concepto "Matritense", que incluiría en uno de sus tres estadios el Solutrense (Pérez de Barradas 1934 a). Esta actividad se complementó con la revisión de antiguas colecciones (Pérez de Barradas 1941). 
Sin embargo, gradualmente fue derivando hacia otros campos de investigación, dejando sin finalizar sus trabajos y sumiendo la investigación de la Prehistoria madrileña en un nuevo letargo (Santonja et al. 2011). No obstante, su clasificación pervivió hasta los años 70 e influyó de forma significativa en el estudio del Solutrense madrileño.

Simultáneamente, los geólogo-paleontólogos, E. Hernández Pacheco (1872-1965) y J. Royo y Gómez (18951961), reinterpretaron la visión de Casiano de Prado acerca del Cuaternario (Royo Gómez y Menéndez 1929). Propusieron unos modelos de terraza (Manzanares, Henares y Jarama) más próximos a los actuales, aunque su influencia fue muy escasa (Santonja et al. 2011).

El reducido número de publicaciones generadas dio poco valor a los estudios realizados antes de 1936, si bien los yacimientos del Manzanares mantenían cierta importancia (Santonja et al. 2011). En este panorama resaltó la figura de J. Martínez de Santa Olalla, quien en 1953 fue nombrado director vitalicio del Servicio de Investigaciones Prehistóricas (Quero 2002; Ortega y Quero 2002). Éste controló los trabajos de campo y la difusión de la parca actividad investigadora. Fruto de ello, fue la publicación de un manual sobre la Prehistoria y la Arqueología nacional (Martínez de Santa Olalla 1941), donde simplificaba las hipótesis de Pérez de Barradas y sustituía algunos términos (Santonja et al. 2011).

Según fue disminuyendo su influencia pasó a realizar recogidas de materiales -tanto industria como fauna-, que produjeron una importante acumulación de piezas tanto en el Instituto Municipal como en el Museo Arqueológico Nacional, M.A.N. (Ortega y Quero 2002; Santonja et al. 2011: 47). Se trataba, en la mayoría de casos, de restos desprovistos de contexto, estudiados recientemente (Baena et al. 2006). Destacan la excavación de Orcasitas y las extracciones controladas en la cantera de Transfesa (Villaverde Bajo) (Santonja et al. 2011: 47).

En la década de 1950 el interés internacional por el estudio del Paleolítico madrileño quedó confirmado con la celebración del IV Congreso Internacional de Ciencias Prehistóricas (1954) y del V congreso del INQUA (1957). El resultado fue la aparición de varios trabajos (Pericot 1952: 44; Pericot 1954) y, sobre todo, la de un libro guía del V Congreso del INQUA (Riba 1957), donde se hacía una síntesis de los nuevos trabajos de campo, actualizando los datos sobre las terrazas del Manzanares. Quedó evidenciada la influencia del esquema planteado por Royo, aunque se mantenía la cronología que Pérez de Barradas daba a las industrias. (Santonja et al. 2011: 47-48).

Pero los modernos métodos de trabajo aparecidos en Europa y África, durante la segunda mitad del s. XX, no fueron utilizados en dichos estudios. Salvando el caso de los realizados, más tarde, por el geólogo Jean Vaudour en lo referente a la evolución del paisaje en la zona de Madrid (Santonja et al. 2011: 49).
El impulso científico iniciado por la Facultad de Ciencias Geológicas, en Madrid, permitió la equiparación de esta disciplina a niveles europeos. Se realizaron nuevas hojas geológicas, a través del Instituto Geológico de España, (Baena et al. 2000), pudiendo reconocerse la complejidad de los valles madrileños y el verdadero número de niveles de terraza (Pérez-González et al. 1974). Observándose, también, fenómenos de hundimiento en diferentes áreas de los ríos Jarama y Manzanares (Pérez-González 1971), lo que habría determinado el aspecto que presentan las estratigrafías de dichos valles (Santonja et al. 2011: 52).

Las nuevas técnicas arqueológicas empleadas en Europa para el Paleolítico medio y superior tardaron algún tiempo en llegar a Madrid (Santonja y Vega 2002). El primer intento fue realizado por Freeman (1975) quien, en la parte destinada al Manzanares, hizo una revisión general del panorama, inmóvil desde 1936 (Santonja et al. 2011), utilizando datos obsoletos y obviando la nueva información geológica.

Finalmente, la nueva metodología se introdujo plenamente a partir de la excavación de Áridos, 1976, en el valle del río Jarama, y de Arriaga, 1981 (Rubio et al. 2002). De forma gradual estos trabajos evolucionaron, apareciendo publicaciones multidisciplinares de investigadores, en su mayoría, vinculados a la Universidad Complutense de Ma-

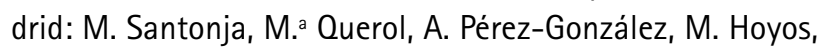
E. Soto y C. Sesé, I. Rus, J. Enamorado, M. Gamazo, A. Cobo, A. Merlo, J. Sánchez etc. (Rubio et al. 2002).

A los trabajos antes mencionados les siguió un pequeño paréntesis, pero la actuación de algunos investigadores particulares y la aparición de la Dirección General de Patrimonio Histórico de la Comunidad de Madrid volvieron a reactivar el trabajo. La remodelación de la M-30, a partir del 2003, provocó la creación de una oficina técnica destinada a evaluar e inspeccionar dichas obras (Santonja 2011). Así una metódica revisión de los valles del Manzanares y Jarama ha aumentado el número de yacimientos pertenecientes a las diferentes fases del Pleistoceno (Gil Ortiz y Calleja 2009; Tapias et al. 2011 a y b; Vega et al. 2011, etc.).

\section{PRESENTACIÓN DE DATOS. EL SOLUTRENSE EN LA COMUNIDAD DE MADRID}

La investigación del Solutrense en España se inició en la segunda mitad del s. XIX, aunque el verdadero impulso se produjo a partir del s. XX con la creación de la CIPP. En esos momentos la investigación estaba muy condicionada por las directrices francesas y destacaban los trabajos de Breuil, Obermaier, Vega del Sella, Hernández Pacheco, Carballo, Aranzadi, Barandiarán, etc (de la Rasilla 1994).

Tras el parón de la Guerra Civil, el reinició de los trabajos se produjo en los años 40, dominado por la teoría del origen africano del Solutrense destacando las figuras de 
Pericot y Barandiaran. Pero el verdadero replanteamiento del periodo se produjo a partir de la década de los 50, cuando se acabó de definir el Solutrense y se asumieron los planteamientos internacionales, pese a contribuciones particulares, destacando los trabajos de Francisco Jordá (de la Rasilla 1994).

Desde los años 70 van a sucederse varios estudios sintéticos sobre el Solutrense (Corchón 1971; Fullola 1979; Straus 1983; Villaverde 1979), junto con monografías de yacimientos en cuya secuencia había niveles solutrenses (Fortea y Jordá 1976; Corchón 1981; Soler y Maroto 1987; Straus 1986; Utrilla 1992; Ripoll 1986; Rasilla 1990). Estas publicaciones fueron consecuencia de la excavación de varios yacimientos (Las Caldas, La Viña, Llonín, Amalda, Abauntz, Chaves, Caldeirão, etc.), así como de la re-excavación de otros (Cueto de la Mina, La Riera, L'Arbreda, Mallaetes, Cueva Ambrosio, etc.) y definen las características del Solutrense peninsular (de la Rasilla 1994).

Así, el Solutrense se inserta dentro de una etapa avanzada del Paleolítico superior, entre el Gravetiense y el Magdaleniense (Corchón 1971: 16). Está dividido en tres periodos, el inicial caracterizado por la presencia de puntas unifaciales, el medio donde destacan las hojas de laurel y puntas de muesca y, por último, el superior donde destacan las puntas de muesca de origen perigordiense (Baena y Carrión 2002). Esta división tipológica, con ciertas matizaciones para la península (Jordá 1957), es la más seguida bibliográficamente, aunque ha recibido fuertes críticas (Straus 1983) y caracterizaciones regionales. En la actualidad, el estudio del Solutrense está dominado por corrientes más tecnológicas, basadas en cadenas operativas (Geneste 1991).

El estudio del registro fósil permite asegurar que la cultura solutrense, en la Península Ibérica, no se presenta de forma homogénea, aparece en grandes zonas, esencialmente, Cantabria y Pirineos occidentales, Cataluña y la base oriental de los Pirineos, grupo atlántico-levantino y, más escasamente, en la zona central (Corchón 1971: 18).

Esta última zona centrará nuestro trabajo, pese a que sus publicaciones y hallazgos son escasos, la mayor parte de los yacimientos del Paleolítico superior en el centro peninsular, Mucientes (Martín et al. 1986), Verdelpino (Moure y Fernández-Miranda 1977), La Hoz y Los Casares (Barandiaran 1973), han sido asignados a fases evolucionadas de este momento: Solutrense y Magdaleniense. Por tanto el Solutrense de la zona supone un "oasis" respecto al resto de manifestaciones.

A finales del s. XIX se referenció el yacimiento de Valdocarros (Vilanova 1891), señalándolo como el primer conjunto solutrense de la zona, y evidenciándose los escasos hallazgos y el desconocimiento sobre esta expresión cultural, en comparación con otras zonas. Posteriormente, Louis Siret referenció otra pieza solutrense en San Isidro (Puche Riart 2008), lo que marcó el devenir del estudio sobre el Solutrense en la región.
Hoy dia hay numerosos restos atribuidos a este tecnocomplejo en los museos, fruto de recogidas antiguas y, generalmente, con precaria caracterización geo-arqueológica, dificultándose así su atribución cultural por: su temprano descubrimiento; la cantidad de materiales; la pérdida de algunos de ellos; y paradigmas explicativos ya descartados; etc. Problemáticas estas que aparecen reflejadas en la bibliografía paleolitista madrileña (Bernaldo de Quirós y Cabrera 1979) y que pasaremos a desarrollar en la discusión.

Los hallazgos atribuidos al Solutrense presentan una distribución dispar a lo largo de la Comunidad de Madrid, muy apreciable entre las áreas de la sierra y las cuencas sedimentarias de los principales ríos. Se observa, a su vez, una sobredimensión, afianzada por el desarrollo urbano de unas cuencas fluviales sobre otras, Manzanares y Jarama sobre Tajuña o Guadarrama (Baena y Carrión 2006). Por tanto, las principales series de terrazas concuerdan con las zonas arqueológicamente más ricas, los valles del Manzanares y el Jarama (Baena et al. 2000).

El Manzanares presenta 12 terrazas escalonadas a las que se tiene que unir la terraza compleja Butarque (PérezGonzález 1994; Pérez-González y Uribelarrea 2002; Silva et al. 2012). De dichos niveles, los situados a $+8 m_{1}+10 m, y+$ 12-15 $\mathrm{m}$ son los relativos al Pleistoceno superior avanzado, aunque no se conserva la serie completa por diferentes fenómenos estructurales (Baena et al. 2000). En los tres niveles asociados al Paleolítico superior se han hallado importantes concentraciones de materiales solutrenses. De esta larga lista se pueden destacar: El Sotillo, Las Delicias, La Carolina, Tejar del Portazgo, Casa del Moreno, Huerto de San Andrés, Prado de los Laneros, Atajillo, López Cañamero, La Parra, o la Fuente de la Bruja, etc. (Baena y Carrión 2002,). Completan la lista los materiales pertenecientes a la Colección Bento, que hoy día se encuentran en el Museo de Arqueología de Cataluña (Barcelona), pero cuya procedencia se encuentra en las recogidas efectuadas en la cuenca del Manzanares: Martínez, Nicasio Poyato, El cojo, Valdivia, Santiago y Prado de la Sociedad de Ganaderos, etc. (Blasco y Carrión 2002).

Sobre muchos de estos yacimientos pesa su temprano hallazgo (Pérez de Barradas 1926; Wernert y Pérez de Barradas 1925) y las condiciones que rodearon a su descubrimiento y posterior adscripción cultural, pues han sido atribuidos a periodos culturales que la ciencia prehistórica, hoy, descarta. Los utensilios aparecieron en terrazas fluviales, en muchos casos mezclados con herramientas del Paleolítico antiguo o localizados en posiciones dificiles de comprender, según los conocimientos geológicos del momento (Santonja y Vega 2002). Todo ello dio lugar a que fueran interpretados dentro de facies irreales y bajo teorias difusionistas, añadiéndose una limitación más debido a la forma en la cual se realizó su recogida. Sin embargo no por ello son desdeñables a nivel arqueológico (Bernaldo de Quirós y Cabrera 1979).

Podemos dividirlos en función de su calidad, dando una mayor validez a los datos de Pérez de Barradas, Obermaier 
y Wernert sobre aquellos pertenecientes a colecciones anteriores o sobre aquellos obtenidos después de la Guerra civil. El motivo fue la exhaustividad con la que realizaron los trabajos y las anotaciones que hicieron acerca de las posiciones estratigráficas y de sus localizaciones (Bernaldo de Quirós y Cabrera 1979). De entre todas estas referencias, hay dos yacimientos que destacan por encima de todas, los yacimientos de El Sotillo y Las Delicias.

El Sotillo es un yacimiento que se localizaba cerca de la actual calle de Antonio López, el hallazgo y las excavaciones se produjeron durante los primitivos trabajos de Wernert y Pérez de Barradas (Martínez de Merlo 1994). Se trata de un yacimiento ampliamente referenciado y que a lo largo de la bibliografía ha sido atribuido a diferentes periodos culturales, Musteriense de influencia africana (Pérez de Barradas 1922), Precapsiense (Obermaier y Pérez de Barradas 1924), Musteriense iberomauritano (Pérez de Barradas y Wernert 1930), Matritense (Pérez de Barradas 1934b), Musteriense iberomauritanico, Esbaikiense (Martínez de Santa-Olalla 1946) etc.

Tras un amplio estudio tipológico y geoestratigráfico que lo asoció a la terraza de $+8 \mathrm{~m}$ del Manzanares parece clara una adscripción finipleistocena, concediendo para algunas de las series ( $B$ y C) una clara vinculación al Solutrense medio (Martínez de Merlo 1994: 65-68). Entre las características que presenta la industria de dicho yacimiento, destaca la abundancia de útiles retocados, atribuidos al Paleolítico superior (raspadores poco diversificados, buriles diedros, de ángulo y sobre truncadura) y, especialmente, de hojas de laurel (de aspecto basto o, tal vez en fases de trabajo) de base convexa y posiblemente biapuntadas (Martínez de Merlo 1994). Pese a la ausencia de puntas de cara plana y de industria ósea, las revisiones más recientes permiten atribuirla definitivamente al Solutrense (Baena y Carrión 2006, Baena et al. 2000).

El yacimiento de Las Delicias fue descubierto por Alejandro Guinea, en 1911, y excavado a partir de 1917 por Obermaier y Wernert. En el lugar que hoy ocupa el Museo y la estación del ferrocarril de dicho nombre (Obermaier y Wernert 1818). Estaba situado en el interfluvio Manzanares/ Arroyo del Hospital (sobre una plataforma a unos 35/40 m. sobre el río Manzanares), en un entorno de transporte de escasa energía, propio de una pequeña vaguada endorreica que filtraba hacia el Manzanares (Santonja et al. 2000). El yacimiento, tras la excavación y los primeros trabajos, recibió sucesivas reinterpretaciones que lo han convertido en uno de los yacimientos de mayor importancia de la Comunidad de Madrid, interés mantenido hasta la actualidad.

En un principio fue vinculado al Paleolítico inferior, filiación más aceptada durante todo este tiempo, descartando la posibilidad de que perteneciera al Paleolítico superior (Obermaier y Wernert 1918: 31-33). Después fue definido como Musteriense de tradición Achelense e influencia Esbaikiense (Obermaier y Pérez de Barradas 1924), como Achelense supe- rior (Royo et al. 1929), o Achelense final, el Achelense VIII de Breuil (Pérez de Barradas 1934 a). Ya en épocas más recientes, finales de la década de los 80, fue entendido como Presolutrense y asociado a un Musteriense evolucionado afín, quizás, con las industrias foliáceas centroeuropeas (Jordá 1986), pero la discusión seguia, pues otros investigadores iban más allá y apuntaban a una industria solutrense (Baena y Carrión 2006; Baena et al. 2000). No obstante, la idea más extendida seguía manteniendo una adscripción Achelense (Santonja et al. 2000).

Las nuevas revisiones del material desde una perspectiva tecnológica (Baena y Carrión 2002; 2006; Baena et al. 2000) y nuevas excavaciones (diciembre de 2008 y julio de 2009) realizadas sobre depósitos aún intactos en las inmediaciones del yacimiento parecen apuntar hacia una industria que, tecnológicamente, confirmaría la hipótesis solutrense (Santonja et al. 2011).

Los nuevos estudios apuntan a la presencia de industria con características similares a las que presenta el grupo clásico manzanareño (el Sotillo o Valdivia), con útiles en diferentes fases de fabricación, lo que pudo haber llevado a error a la hora de interpretar como elementos terminados lo que en realidad eran piezas en proceso de reducción bifacial (Baena y Carrión 2002; 2006; Baena et al. 2000). La presentación final de los resultados de estos nuevos trabajos podrá apoyar esta hipótesis.

También, hemos de mencionar el yacimiento de Las Carolinas. Fue excavado en 1911 por D. Alejandro Guinea y, en 1916, por Obermaier, quien posteriormente dio a conocer los resultados (Obermaier y Wernert 1917). Se situaba en el margen derecho de la carretera Madrid-Andalucia (término municipal de Madrid), a unos $200 \mathrm{~m}$ del margen derecho del río Manzanares y frente a la confluencia de éste con el arroyo Abroñigal (Blasco y Baena 1996). El desarrollo de las dos campañas acabó con un resultado desigual, mientras que Obermaier tan sólo localizó restos paleolíticos poco significativos, Guinea encontró varios niveles, en los superiores habia restos de un yacimiento campaniforme $y$, bajo él materiales paleolíticos (Obermaier y Wernert 1917; Blasco y Baena 1996). Sin embargo, es complicado pronunciarnos sobre las características de la industria, por la pérdida de parte de los materiales y la carencia de datos referentes al contexto en que se encontraban. A todo ello hay que sumar la falta del dibujo de las piezas. Pese a ello se ha apuntado al Paleolítico superior (Blasco y Baena 1996; Baena y Carrión 2006; Baena et al. 2000;) e incluso al Solutrense (Gamazo 2002) como filiación más plausible, idea que, por la proximidad a la concentración de los restos ya comentados, no sería descartable.

Las intervenciones realizadas en la M-30, a partir del 2002-2003, sobre las inmediaciones del arroyo Abroñigal, un afluente del Manzanares, han dado como resultado la aparición del yacimiento del Puente de los Tres Ojos. Conjunto Musteriense en posición derivada, aunque en la misma intervención aparecieron varias piezas correspondientes a 
modos de producción laminar (un núcleo y algunas láminas), así como fragmentos de puntas foliáceas solutrenses con retoque total (muy profundo), plano que hacen pensar en su pertenencia al Solutrense (Tapias et al. 2011 b: 279).

También son reseñables algunas lascas con retoques invasores relacionables con la fabricación de piezas foliáceas solutrenses halladas en un nivel de terraza $+5-8 \mathrm{~m}$. del arroyo Abroñigal a la altura de la A-3 (Tapias et al. 2011a). El estudio de dichos yacimientos, así como el hallazgo de una hoja de laurel en el nivel $+8 \mathrm{~m}$ de la terraza del Manzanares, aguas arriba del Estanque de Tormentas de Butarque (Gil Ortiz y Calleja de Dios 2009) podría suponer una ampliación de los conjuntos Solutrenses del Manzanares.

En la zona del valle del Jarama, y sus afluentes, también resulta difícil identificar la secuencia completa de terrazas, pero a lo largo de su recorrido se han podido identificar nueve plataformas (Pérez-González y Calvo 1989), de las que corresponden a momentos avanzados del Pleistoceno las de $+8 m+12-15 m$ (Baena y Carrión 2002; 2006).

En la cabecera del Jarama y el río Sorbe destacan el Abrigo de Peña Capón y el Abrigo de Peña Cabra. Este último está situado sobre una ocupación del Paleolítico medio, que cuenta con industria entre la cual destacan elementos foliáceos y plaquetas de pizarra con líneas incisas (García Valero 2002). Estas características nos descubren "un posible horizonte Solutrense" (Baena y Carrión 2006).

Aunque sin duda, el Abrigo de Peña Capón es donde más claramente se puede hablar de Solutrense medio (Alcolea et al. 1997; García Valero 2002). Situado en el término municipal de Muriel (Guadalajara), los trabajos arqueológicos se iniciaron a principios de los años 70 bajo la dirección de Julio Martínez Santa-Olalla (Alcolea et al. 1997; Cacho, 2010; García Valero 2002). Presenta varios niveles arqueológicos, el 3 adscrito a principios del Paleolítico superior aunque con dudas, el nivel 2 asignado al Solutrense, y sobre ellos un nivel de cronología no definida (quizá holocena) (Alcolea et al. 1997; Cacho et al. 2010; García Valero 2002).

En el nivel 2 de Peña Capón apareció un conjunto de elementos foliáceos bifaciales, donde destacan las hojas de laurel de base convexa, las hojas de sauce y algunos tipos (puntas asimétricas bifaciales) que los autores relacionan con prototipos franceses (Alcolea et al. 1997). A pesar de la presencia de elementos de cara plana, estas características hacen viable una interpretación como Solutrense medio (Alcolea et al. 1997; Cacho et al. 2010; García Valero 2002).

También en el Jarama, en su tramo medio, aparecen hallazgos más escuetos como los de Casa Garcini (San Fernando de Henares) (Baena et al. 2002) y sobre todo Valdocarros. La industria de Valdocarros fue cedida a Vilanova a finales del s. XIX (Vilanova 1891) y significó la primera referencia del Solutrense en la zona centro. El material no tiene una ubicación determinada, se sabe que procede del despoblado de Valdocarros (finca "El Piul"), Arganda del Rey, en el margen derecho del antiguo cauce del Jarama (Blasco et al. 1984; Vilanova 1891).

Se trata de un reducido número de piezas, que tras varios análisis es posible asociar al Solutrense (Baena y Carrión 2006; Blasco et al. 1984; 1998; Vilanova 1891), algunas de ellas de evidente caracterización solutrense, destacando una pieza foliácea (Fig. 1) de morfología semejante a las hojas de laurel que posiblemente se fracturó por flexión (Vilanova 1891; Blasco et al. 1984).

Las mencionadas actuaciones que se dieron a raíz de los trabajos de la M-30 también han tenido su relevancia en la zona del Jarama, así podemos apuntar la posible ampliación del material perteneciente al Paleolítico superior (Tapias et al. 2011 b; Vega et al. 2011). Su estudio detallado podrá revelarnos que incluso parte pertenezca al Solutrense.

Fuera de la Comunidad de Madrid, en el norte, hay que referenciar yacimientos descontextualizados pertenecientes al Solutrense o Magdaleniense inicial. Destaca Barranco Hondo, en el término de Villalba, con evidencias de arte mueble (Baena et al. 2006), aunque de cronología discutida. También, en los límites de la Meseta sur hemos de citar el Abrigo del Palomar, situado en las estribaciones de la Sierra de Alcaraz (Albacete). En el nivel III han aparecido útiles característicos del Solutrense, una hoja y elementos de aleta y pedúnculo realizados mediante retoque total (muy profundo), raspadores con retoque plano, etc. Se trata de un yacimiento cuyo estudio puede ser importante para dilucidar las relaciones de contacto del Mediterráneo y la Meseta (Cacho et al. 2010).

En su conjunto, los datos expuestos permiten aceptar la ocupación solutrense del interior peninsular, tradicionalmente considerado inhabitado, o sólo frecuentado esporádicamente. Los útiles muestran ciertas peculiaridades como piezas de mayor tamaño (hojas de laurel) y la escasez de puntas de muesca de tradición Gravetiense, si bien presentan otros elementos más comunes de las áreas levantina y portuguesa, como los pedúnculos (puntas romboidales con pedúnculo incipiente) (Baena y Carrión 2006; Corchón, 1971). La talla bifacial es la más representada obteniéndose piezas laminares de dimensiones notables que dan lugar a vestigios tipológicamente grandes, hojas de laurel y sauce, raspadores, raederas, buriles diedros y sobre truncadura, etc. Estas características han podido sugerir que los elementos en distintos estadios de fabricación pueden haber contribuido a interpretar como elementos finales piezas en proceso de reducción bifacial (Baena y Carrión 2006; Baena et al. 2000).

Estas características tecno-tipológicas permiten encuadrar gran parte de las industrias solutrenses meseteñas en momentos del Solutrense medio y superior, si atendemos a la secuencia propuesta para el tecnocomplejo en diversas regiones peninsulares y francesas (Rasilla 1994). 


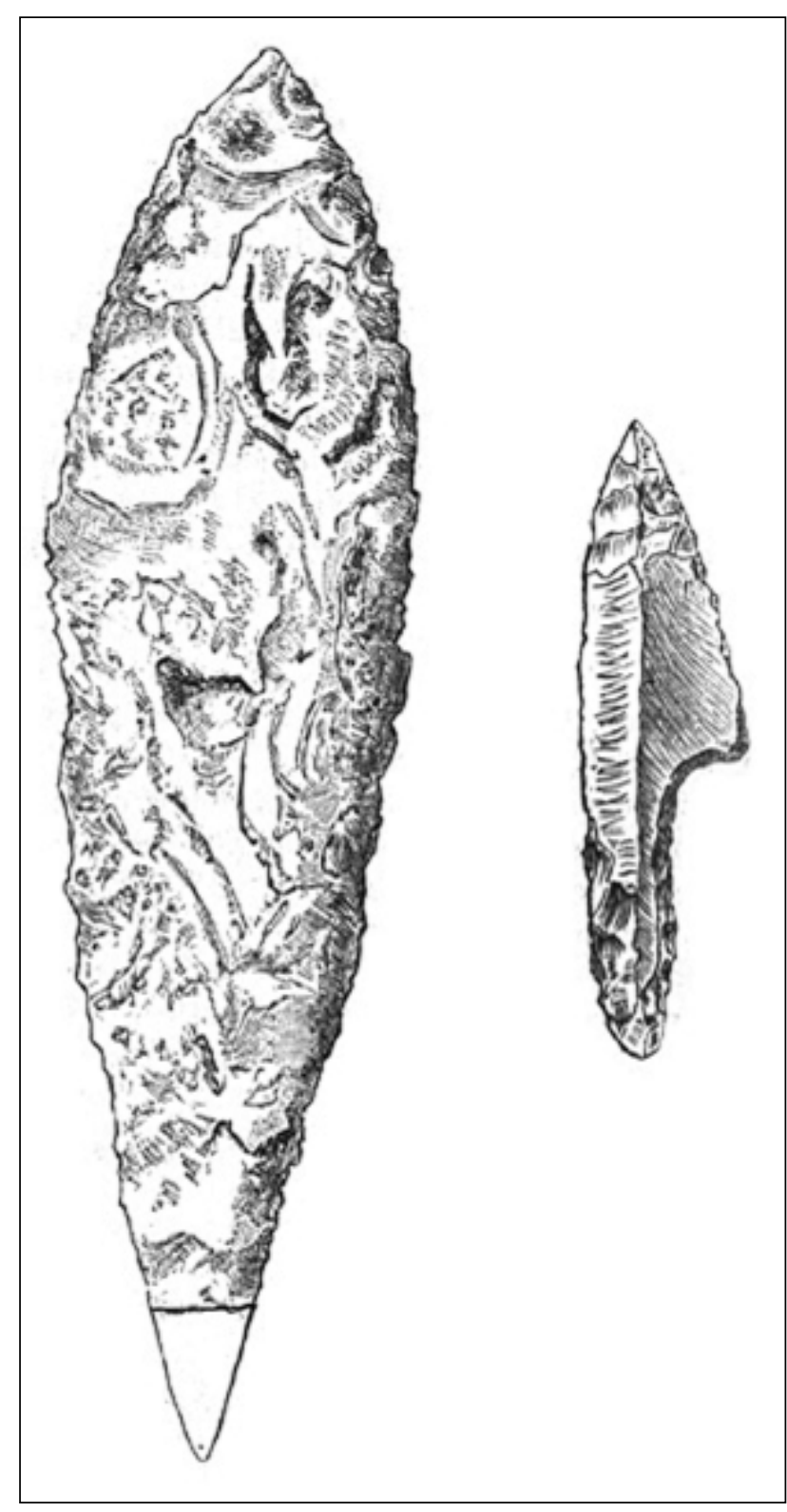

ム Figura 1. Piezas solutrenses de Valdocarros (Vilanova 1981).

\section{PROBLEMÁTICA Y DISCUSIÓN}

La revisión historiográfica demuestra que hay una abundante cantidad de materiales paleolíticos en la Comunidad de Madrid pero, en muchos casos, su atribución cultural ha resultado muy compleja debido a las condiciones de su recuperación. Por ello se dan numerosas dificultades en la definición del Solutrense en esta área geográfica, tal y como se apunta en los párrafos anteriores. Sin embargo sería conveniente explicarlas de forma más detenida.

El temprano hallazgo de los yacimientos y las técnicas utilizadas en la recuperación del registro afectan a su adscripción cultural. La caracterización y comprensión de los restos solutrenses han estado marcadas por la complejidad que presentan las terrazas de los valles del Manzanares y el
Jarama, de ahí la incorrecta filiación cultural de los materiales. Son depósitos de terraza complejos ya en su génesis que, posteriormente, sufrieron procesos de solapamiento, erosión y redeposición de materiales. Además la tectónica cuaternaria y sus consecuencias (fallas, paleoseísmos, etc.) han provocado la existencia de coluviones y aportes laterales posteriores y fenómenos de solifluxión y crioturbación. (Pérez-González, 1971, Pérez-González et al. 1974; Silva et al. 2012). Todo ello ha motivado la confusión en el estudio e interpretación del registro arqueológico, pues el Paleolítico superior podría encontrarse en coluviones más recientes 0 en cualquier nivel de terraza, provocando mezclas en el registro fósil (Silva et al. 2012).

La desaparición de la mayoría de yacimientos debido al posterior crecimiento de la ciudad y sus infraestructuras supone otro problema en el posterior registro de los materiales. La falta de control de la posición de los mismos en la estratigrafía y su recogida, especialmente en el caso de los de pequeño formato, supuso una importante pérdida de datos (Bernaldo de Quirós y Cabrera 1979).

Otro inconveniente, que exige una amplia reflexión, de cara a la correcta interpretación de los conjuntos madrileños, es la terminología empleada, especialmente por Pérez de Barradas (1924) y Martínez Santa-Olalla (1946). El primero cita niveles del Musteriense denominados Esbaikienses o de influencia africana, incluyendo el famoso Musteriense iberomauritánico. Entre los niveles musterienses habla del término Musteriense de tradición achelense (diferente al de F. Bordes) al que pertenecian estos niveles con presencia de bifaces. De este modo se puede considerar que la aparición de bifaces no es pertinente, pues podría pensarse en materiales rodados o heredados. Si nos centramos en el término Esbaikiense, definido por la presencia de "puntas tennuifoliadas" (delgadas, talladas y retocados por ambas caras), originarias de la región S'baikiia. (Obermaier 1925: 98). Se trata, en general, de tipos ovales, alargados, puntiagudos en ambos extremos, que recuerdan bastante las formas foliáceas solutrenses.

El Musteriense iberomauritánico resulta también de una mezcla cultural, sólo aparece en el Valle del Manzanares y se identificaría nuevamente por la presencia de puntas tenuifoliadas "sbaikienses" y tipos evolucionados (buriles y raspadores), en este caso influidos por el Ateriense africano (Obermaier 1925). Fueron mencionados en yacimientos (EI Sotillo) donde aparecen unidos estos términos (Musteriense Clásico, Sbaikiense y Ateriense), lo que hizo pensar que eran modalidades de una nueva facies, Musteriense iberomauritánico (Obermaier 1925: 227).

Pérez de Barradas (1934 a) introdujo un nuevo término, Matritense, que posteriormente desarrolló Martínez SantaOlalla. Pérez de Barradas lo utiliza para definir una fase local del Paleolítico superior, en la cual estaría el Solutrense. Martínez de Santa-Olalla lo refiere al Paleolítico medio, con dos 
fases, la I incluiría el Sbaikiense y la II incluiria grandes "hachas de corte transversal talladas sobre lasca levalloisiense". El Paleolítico superior del Manzanares aparece bajo la forma del Matritense III "con piezas de estilo Ateriense y otras de aire marcadamente presolutrense" (Martínez Santa-Olalla 1946). La definición de estas industrias parte de conceptos, Ateriense y Sbaikiense, y relaciones (África-España) ya superados, lo que ha provocado una amplia revisión y lectura de las estratigrafías para solucionar malentendidos y conceptos erróneos.

Expuestos todos estos inconvenientes se pondrian encontrar piezas arcaicas junto a modernas, explicando así la existencia de estos "Musterienses de tradición achelense" que se podrian convertir en el Musteriense, propiamente dicho, o incluso en un Paleolítico superior. Hay que destacar que estas piezas bifaciales no tienen obligatoriamente que presentar un rodamiento excesivo. El hundimiento de materiales puede hacer que las piezas procedentes de niveles altos de terraza resbalen o caigan de los cortes entrando a formar parte del coluvión sin haber apenas rodado, por lo que se conservarán frescas y sin pátina especial, si no es por la edáfica que tendrán las demás piezas.

La muestra de restos solutrenses descrita anteriormente, con las limitaciones mencionadas, puede dividirse en función de la calidad de los registros conservados. Así las exhaustivas anotaciones de Pérez de Barradas, Obermaier y Wernert, que señalan las posiciones estratigráficas donde aparecía la industria merecen tenerse en cuenta, a pesar de que proceden de recogidas superficiales o de excavaciones realizadas con la metodología de los años veinte (El Almendro y El Sotillo, etc.) (Martínez de Merlo 1984). En otros casos, las referencias bibliográficas aparecen apoyadas en documentación que hace más factible hablar de un horizonte Solutrense en los areneros de Valdivia, Martínez, Nicasio Poyato y El Cojo, Prado de los Ganaderos (Baena y Carrión 2002).

Es significativa la alta densidad de hallazgos en una zona restringida del Manzanares, interpretada como un área, tal vez, de captación de este entorno durante el Paleolítico superior (Baena y Carrión 2006: 48), aunque también puede deberse a la importante explotación que desde el s. XIX sufrieron los areneros de áridos, hecho que ha permitido una mayor visibilidad de materiales.

Junto a las referencias que datan del siglo pasado, hemos hallado evidencias más recientes, ya sean aquellas que complementan superficies antes estudiadas (Delicias) o que tratan nuevos hallazgos (El abrigo de Peña Capón, Puente de los tres ojos, etc.,). Todo esto hace posible hablar de una importante concentración de restos asignables al Solutrense medio y superior en la zona centro de la península, en concreto en la Comunidad de Madrid, y nos permite ser optimistas en cuanto al futuro desarrollo de los estudios sobre el Solutrense. No obstante, debemos puntualizar que hasta fechas no muy lejanas no hubo especialistas en Madrid dedicados al Solutrense, siendo una época más es- tudiada en otras regiones. Por lo general las investigaciones realizadas en el centro peninsular se dedicaban a los periodos más antiguos del Paleolítico.

\section{CONCLUSIONES}

Las referencias materiales y bibliográficas demuestran, como se ha venido comentando, que la investigación del Paleolítico en Madrid data de muy antiguo, sin embargo ha pasado por varias fases de letargo o casi olvido.

En el Solutrense hay que indicar que sus evidencias son muy abundantes, procediendo, la mayoria, de las terrazas de los valles del Manzanares (El Sotillo, Las Delicias, Puente de los tres ojos, Las Carolinas, Tejar del Portazgo, Casa del Moreno, Huerto de San Andrés, Prado de los Laneros, Atajillo, López Cañamero, La Parra, La Fuente de la Bruja, o los yacimientos que componen la Colección Bento, etc.) y, en menor medida, del Jarama (Peña Capón, Valdocarros, etc).

Muchos de los antiguos yacimientos, tras revisiones tecnológicas con metodologías actuales, nuevos estudios geoarqueológicos de las terrazas en que se hallaban y la excavación de terrenos colindantes a los inicialmente excavados, presentan una adscripción solutrense. Entre todos destacan los yacimientos de El Sotillo, Las Delicias, Valdocarros, el Abrigo de Peña Capón, la colección Bento etc. Además, la aparición de nuevas evidencias solutrenses, aún en fase de estudio, con motivo de la remodelación de la M-30, permiten aceptar la existencia de ocupaciones solutrense en zonas del interior peninsular.

La historiografía de la Comunidad de Madrid, y de la península, en el pasado mencionaba los escasos restos solutrenses de la zona, para explicar áreas de influencia o de comunicación entre unas $u$ otras franjas de la península 0 como una fase intermedia en la creación de variantes regionales (Corchón 1971; Straus 1983). Hoy, parece claro que esto no es así o por lo menos no en todos sus componentes, siendo factible que el Solutrense de la zona centro tenga su propia entidad e independencia y no hiciera referencia a ocupaciones esporádicas. Entre los rasgos que permitirian definir esta nueva situación observamos el mayor tamaño de las piezas.

El estudio del Paleolítico madrileño, tradicionalmente, se ha decantado por la investigación de los periodos más antiguos y desatendiendo de alguna manera los restos pertenecientes al Paleolítico superior (Bernaldo de Quirós y Cabrera 1979). De esta forma los materiales de menor tamaño, precisamente los más abundantes en el Paleolítico superior, han sido despreciados y desaprovechados para la investigación, y, otras veces, la presencia de materiales pertenecientes a conjuntos mezclados acabó recibiendo una única atribución, la más antigua. Hoy parece que ese panorama está cambiando, así aparecen investigadores dedicados al 
estudio del Paleolítico superior en general y del Solutrense en particular. Esta nueva tendencia puede deparar sorpresas positivas, primero aumentando el número de yacimientos solutrenses de la Comunidad de Madrid y, después, completando la investigación y caracterización de los ya existentes.

Uno de los retos que deben afrontar los nuevos investigadores y especialistas, en la zona centro y especialmente en Madrid, es poner fin al dispar estudio de las áreas territoriales, pues como se ha apuntado anteriormente existe por costumbre o por connotaciones de explotación residencial y económica, un mayor interés o trabajo en las zonas fluvia- les de los grandes ríos - Manzanares y Jarama-, quedando abandonadas los terrenos de montaña, como los que componen las sierras de Madrid. Aunque no por ello deben olvidarse las cuencas secundarias, que tampoco han sido objeto de grandes hallazgos.

Sin embargo, un nuevo problema parece presentarse, pues con la agudización de la crisis económica se está iniciando un proceso de paralización de las obras y con ello de los trabajos arqueológicos. Esto puede provocar una nueva fase de letargo en la investigación del Paleolítico madrileño, y por ende del Solutrense de la zona centro peninsular.

\section{BIBLIOGRAFÍA}

Alcolea, J., Balbín, R., Garcia, M. A., Jiménez, P. J., AldecoA, A., Casado, B., De Andrés, B., Ruiz, S., SaInz, P. y SúAReZ, N. 1997: "Avanze al estudio del poblamiento paleolítico del Alto Valle del Sorbe (Muriel Guadalajara)". Il congreso de Arqueología Peninsular. Zamora: 201-218.

AyarZagüEnA, M. 2002: "Juan Vilanova y Piera". En Panera, J. y Rubio, S. (coords.): Bifaces y Elefantes. La investigación del Paleolítico inferior en Madrid. Zona Arqueológica 1: 56-79.

BAenA, J. y CARRIÓN, E. 2002: "Los materiales solutrenses". En C. Blasco Bosqued: La colección Bento del Museu d'Arqueologia de CataIunya. Una nueva mirada a la Prehistoria Madrileña. Barcelona: 79-130.

- 2006: "Contexto geográfico: Un marco para el desarrollo del Paleolítico superior de Madrid". En M. R. Lucas Pellicer, L. M. Cardito RoIlán y J. Gómez Hernánz (eds.): Dibujos en la Roca: El arte rupuestre en la comunidad de Madrid. Comunidad de Madrid, Consejería de Cultura y Deportes. Madrid: 43-55.

Baena, J., Conde, C., CARRIÓn, E. y Pastor, F. J. 2000: "Paleolítico y Epipaleolítico. En la Arqueología madrileña en el final del siglo XX". Boletín de la Asociación Española de Amigos de la Arqueología 3940: 81-104.

Baena, J., Conde, C., Gamazo, M., Sesé, C., Soto, E. 2002: "Repertorio de yacimientos paleolíticos en el Manzanares y Jarama". En Panera, J. y Rubio, S. (coords.): Bifaces y Elefantes. La investigación del Paleolítico inferior en Madrid. Zona Arqueológica, 1: 460-491.

BaRAndiaran, I. 1973: "La cueva de los Casares (en Riba de Saelices, Guadalajara)". Excavaciones Arqueológicas en España 76. Madrid.

Bernaldo de Quirós, F. y Cabrera, V. 1979: "Problemas generales del Paleolítico Medio y Superior en la provincia de Madrid". I Jornadas de Estudios sobre la provincia de Madrid. Diputación provincial de Madrid. Madrid: 53-56.

Blasco, M. C. y BaENA, J. 1996: "El Yacimiento de las Carolinas y la cerámica simbólica campaniforme. Algunos datos para su interpretación". En A. Moure (ed.): "El hombre fósil. 80 años después: homenaje a Hugo Obermaier". Editorial Universidad de Cantabria. Cantabria.

Blasco, M. C. y CarRión, E. 2002: "La Colección Bento en su marco histórico y geográfico". En Panera, J. y Rubio, S. (coords.): Bifaces y Elefantes. La investigación del Paleolítico inferior en Madrid. Zona Arqueológica 1: 420-429.

Blasco, M. C., BAENA, J. y Von LetTOW-VorbeCK, C. L. 1984: "La prehistoria Madrileña en el Gabinete de Antigüedades de la Real Academia de la Historia. Los yacimientos Cuesta de la Reina (Ciempozuelos) y Valdocarros (Arganda del Rey)". Universidad Autónoma de Madrid. Madrid: 75-117.

BlasCo, M. C., BAENA, J. y LIESAU, C. 1998: "La prehistoria madrileña en el gabinete de antigüedades de la Real Academia de la Historia". Patrimonio Arqueológico del bajo Jarama 3.
Cacho, C. y Martos, J. A 2002: "Colecciones paleolíticas de Madrid en el Museo Arqueologico Nacional". En Panera, J. y Rubio, S. (coords.): Bifaces y Elefantes. La investigación del Paleolitico inferior en Madrid. Zona Arqueológica 1: 382-407.

Cacho, C., Martos, J. A., Jordá Pardo, J., Yravedra, J., Avezuela, B., Valdivia, J. y MARTín, I. 2010: "El Paleolítico superior en el interior de la Peninsula Ibérica. Revisión critica y perspectivas de futuro". En X. Mangado (ed.): El Paleolítico superior peninsular. Novedades del siglo XXI. Homenaje al Profesor Javier Fortea. Monografies 8. SERP. Barcelona: 75-96.

Carrera, E. De y Martin Flores, A. 2002: "José Pérez de Barradas. Una biografia intelectual". En Panera, J. y Rubio, S. (coords.): Bifaces y Elefantes. La investigación del Paleolítico inferior en Madrid. Zona Arqueológica 1: 108-147.

CORCHÓN, M. S. 1971: "El Solutrense en Santander". Instituto de Prehistoria y Arqueología "Sautuola", Institución Cultural de Cantabria. Diputación Provincial de Santander. Santander.

- 1981: "La Cueva de Las Caldas. San Juan de Priorio (Oviedo)". Excavaciones Arqueológicas en España, 115. Ministerio de Cultura. Madrid.

DíAZ-ANDREU, M. 2000: "La arqueología imperialista en España: extranjeros vs. españoles en el estudio del arte prehistórico de principios del siglo XX". En J. Alarco et al. (eds): Historia, Teoría e pratica da Arqueologia. Ass. para o Desenvolvimento da Coop. em Arq. Peninsular. Universidad do Porto. Oporto: 367-382.

FORTEA, J. y JORDÁ, F. (1976): "La Cueva de les Mallaetes y los problemas del Paleolítico Superior del Mediterráneo español", Zephyrvs XXVIXXVII: 129-166.

FreEMAN, L. G. 1975: "Acheulian Sites and Stratigraphy in lberia and the Magreb". En K. W. Butzer y G. Isaac (eds.), After the Australopithecines. Mouton Pub. The Hague-Paris: 661-744.

Fullola, J. M. 1979: Las industrias líticas del Paleolítico superior lbérico. Trabajos Varios 60. Servicio de Investigación Prehistórica. Valencia: $262 \mathrm{p}$.

Gamazo, M. 2002: "Las colecciones paleolíticas del Manzanares y del Jarama del Museo de San Isidro". En Panera, J. y Rubio, S. (coords.): Bifaces y Elefantes. La investigación del Paleolítico inferior en Madrid. Zona Arqueológica 1: 358-381.

Garcia Valero, M. A. 2002: "El Paleolítico en Guadalajara". Actas del primer Simposio de Arqueología de Guadalajara. Sigüenza, 4-7 octubre 2000. 1. Ayuntamiento de Sigüenza: 145-186.

GENESTE, J. M. (1991): "Systèmes techniques de production lithique: variations techno-économiques dans les processus de réalisation des outillages paléolithiques". Techniques et Culture 17-18: 1-35.

GIL ORTIZ, C. y CALLEA, M. 2009: "Resultados del seguimiento geoarqueológico de las obras de construcción del Colector de Margen Derecha. Zona Sur". Actas de las 4 Jornadas de Patrimonio Arqueológico en la Comunidad de Madrid (Alcalá de Henares, 2007). Madrid: 349-352. 
JORDÁ, F. 1957: "La España de los tiempos paleolíticos. Las Raíces de España". Instituto Español de Antropología Aplicada. Madrid: 28 pp.

- 1986: "Paleolítico". En V.V.A.A.: Historia de España, I. (Prehistoria). Editorial Gredos. Madrid: 8-150.

MAIER, J. 2000: "Historiografía de la Arqueología Matritense". En La Arqueología madrileña en el final del siglo XX: desde la Prehistoria hasta el año 2000. Boletín de la Asociación Española de Amigos de la Arqueología 39-40: 41-66.

Martin, E., Rojo, A. y Moreno, M. A. 1986: "Habitat postmusteriense en Mucientes (Valladolid)". Numantia. Investigaciones Arqueológicas en Castilla-León II: 87-99.

Martinez de Merlo, A. M. 1984: "El Paleolítico superior en el valle del Manzanares: el yacimiento de El Sotillo". Boletín del Museo Arqueológico Nacional II (1): 47-68.

Martinez de Santa-Olalla, J. 1946: "Esquema paleontológico de la Península Hispánica". Publicaciones del Seminario de Historia primitiva de Hombre. Madrid.

Moure, J. A. y FernÁndez-MiRAndA, M. 1977: "El abrigo del Verdelpino (Cuenca). Noticias de trabajos de 1976". Trabajos de Prehistoria 34: 31-68.

OBerMaler, H. 1925: "El hombre fósil". 2a edición. Madrid. Comisión de Investigaciones Paleontológicas y Prehistóricas. Memoria, 9. 457 p. (Reedicción fascimilar de la $2^{\mathrm{a}}$ edición: Madrid: Ediciones Istmo. 1985).

Obermaier, H. y Pérez de Barradas, J. 1924: "Las diferentes facies del Musteriense Español y especialmente de los yacimientos madrileños". Revista de la Biblioteca, Archivo y Museo del Ayuntamiento de Madrid I (2): 143-177.

Obermaier, H. y Wernert, P. 1917: "Yacimiento prehistórico de Las Carolinas". Memorias de la Comisión de Investigaciones Paleontológicas y Prehistóricas 16. Madrid: 35 pp.

- 1918: "Yacimiento Paleolítico de Las Delicias (Madrid). Memorias de la real Sociedad Española de Historia Natural, XI. Museo de Ciencias Naturales. Madrid: 5-35.

Ortega, A. I. y Quero, S. 2002: "Julio Martínez Santa-Olalla". En Panera, J. y Rubio, S. (coords.): Bifaces y Elefantes. La investigación del Paleolítico inferior en Madrid. Zona Arqueológica 1: 194-213.

Pérez de Barradas, J. 1922: "Yacimientos paleolíticos del valle del Manzanares. Trabajos de 1920-21". Junta Superior de Excavaciones y Antigüedades, 42. Madrid: 19-27.

- 1924: "Introducción al estudio de la prehistoria madrileña". Revista de la Biblioteca, Archivo y Museo del Ayuntamiento de Madrid 1 (1): 13-35.

- 1926: "Estudios sobre el cuaternario del valle del Manzanares (Madrid)". Imprenta municipal. Madrid.

- 1929: "Los yacimientos prehistóricos de los alrededores de Madrid". Boletin Geologico y Minero LI: 153-322.

- 1933-36: "Nuevos estudios de prehistoria madrileña: La colección Bento". Anuario de Prehistoria Madrileña IV-V (1933-1936): $1-90$.

- 1934 a: "Influences africaines dans le Paléolithique de Madrid". Anuario de Prehistoria Madrileña II-III: 3-11.

- 1934 b: "Los problemas del Paleolítico Superior madrileño". Investigación y Progreso, VIII. Madrid: 249-254.

- 1941: "Nuevas investigaciones sobre el yacimiento de San Isidro (Mádrid)". Archivo español de Arqueología XIV: 277-333.

Perez de BARRAdAs, J. y Wernert, P. 1930: "El yacimiento paleolítico de El Sotillo". Anuario de Prehistoria Madrileña 1: 37-95.

Pérez-GonzÁlez, A. 1971: "Estudio de los procesos de hundimiento en el valle del río Jarama y sus terrazas (nota preliminar)". Estudios geológicos XXXVII: 317-324.

- 1994: Depresión del Tajo. En M., Gutiérrez Elorza, (ed.): Geomorfología de España. Rueda: 389-436.

Pérez Gonzalez, A. y Calvo, J. P. (1989): Hoja y Memoria del 1:50.000 (nr. 559) I.G.M.E. Madrid.

Pérez-GonzÁlez, A. y Uribelarrea, D. 2002: Geología del Cuaternario en los valles fluviales del Jarama y Manzanares en las proximidades de Madrid. En Panera, J. y Rubio, S. (coords.): Bifaces y Elefantes. La investigación del Paleolítico inferior en Madrid. Zona Arqueológica 1: 302-317.

Pérez-González, A., Aleixandre, T., Gallardo, J., Pinilla, A., y Medina, A. 1974: "Valle Henares-Jarama. Guía de la excursión B". Actas de la primera reunión nacional del grupo de trabajo del Cuaternario (G.T.C). Trabajos Neógeno-Cuaternario II: 223-229.

PERICOT, L. 1952: "Las raices de España". CSIC. Madrid.

- 1954: "El Paleolítico y Epipaleolítico en España". IV Cong. Int. de Ciencias Prehist. y Protoh. C.S.I.C. Madrid.

Prado, C. 1864: Descripción física y geológica de la provincia de Madrid. Colegio de Ingenieros de Caminos, Canales y Puertos. Madrid, 1975: 219 p. (1a edición de 1864).

PUCHE RIART, O. 2008: "Historia de la arqueologia madrileña: el proceso del monumento al hombre primitivo en el parque de San Isidro". En 0. Puche Riart (ed.): Documentos inéditos para la Historia de la Arqueología. SEHA, Sociedad Española de Historia de la Arqueología. Memorias de la sociedad española de historia de la Arqueología: 287-297.

QUERO, S. 2002: "La investigación del Paleolítico durante el franquismo (1936-1971)". En Panera, J. y Rubio, S. (coords.): Bifaces y Elefantes. La investigación del Paleolítico inferior en Madrid. Zona Arqueológica 1: 168-193.

RASILLA, M. DE LA 1990: "Cueto de la Mina. Campañas 1981-1986". Excavaciones Arqueológicas en Asturias 1983-1986 1. Consejería de Educación y Cultura. Oviedo: 79-86.

- 1994:"Introducción: El Solutrense en el contexto del Paleolítico Superior Occidental". Férvedes 1: 9-19

RASILLA, M. DE LA y SANTAMARíA, D. 2005: "Algunos promotores de la investigación prehistórica hispana a principios del siglo $X X$, Conde de la vega del Sella, Marqués de Cerralbo, Juan Cabré Aguiló y Amalio Gimeno Cabañas". Archaia 3-4-5: 209-2014.

RoYo GómeZ, J. y MeNÉNDEZ, L. 1929: "Mapa y memoria explicativa de la hoja n. ${ }^{\circ}$ 559, Madrid". Instituto Geológico y Minero Español. Madrid

RoYo GómeZ, J., MENÉNDEZ, L. y ABBAD, M. 1929: "Memoria explicativa de la Hoja Geológica a E. 1:50.000 n. ${ }^{\circ}$ 559. Madrid" Instituto Geológico y Minero. Madrid.

RIBA, 0. 1957: "Terrasses du Manzanares et du Jarama aux environs de Madrid". V Congrès int. de INQUA. Livret-guide de I'Excursion C-2. C.S.I.C. Madrid.

RIPOLL, S. 1986: "El Solutrense de Cueva Ambrosio. Vélez-Blanco, Almeria, campaña de 1963". Excavaciones Arqueológicas en España 148: $205 \mathrm{pp}$.

RubiO, S., Panera, J. y Martos, J. A. 2002: "La modernización del Paleolítico en los valles del Manzanares y Jarama: 1970-1985". En Panera, J. y Rubio, S. (coords.): Bifaces y Elefantes. La investigación del Paleolítico inferior en Madrid. Zona Arqueológica 1: 214-241:

SANTONJA M. 2011: "El esperado reencuentro con el paleolítico Madrileño". En M. Santonja (cord.): Presentación del libro. En M. Santonja (Cord.): Actas de las $5^{a}$ Jornadas de Patrimonio Arqueológico en la Comunidad de Madrid (Los primeros pobladores: Arqueología del Pleistoceno, Alcalá de Henares, 2008). M.A.R. Madrid.

Santonja, M. y Vega L. G. 2002: "La investigación del valle del Manzanares (1862-1975) en el contexto del Paleolítico español". En Panera, J. y Rubio, S. (cords.): Bifaces y Elefantes. La investigación del Paleolítico inferior en Madrid. Zona Arqueológica 1: 242-275.

Santonja, M., Pérez-González A. y Vega L. G. 2000: "El yacimiento de la Estación de Las Delicias (Madrid) y la investigación del Paleolítico en el Manzanares". SPAL 9: 525-555

Santonja M., Pérez González A., Vega L. G. y URibelarrea, D. 2011: "La evolución sobre las ideas sobre el paleolitico en Madrid". En M. Santonja (Cord.): Actas de las $5^{a}$ Jornadas de Patrimonio Arqueológico en la Comunidad de Madrid (Los primeros pobladores: Arqueología del Pleistoceno, Alcalá de Henares, 2008). M.A.R. Madrid: 27-60.

StRaus L. G., 1983: "El Solutrense vasco-cantábrico. Una nueva perspectiva". Centro de Investigación y Museo de Altamira, 10. Ministerio de Cultura. Madrid. 
- 1986: "Late Würm adaptative Systems in Cantabrian Spain: the Case of Eastern Asturias". Journal of Anthropological Archaeology: 330-369

Silva, P. G. M., López Recio, M., Cuartero, F., Baena, J., Tapias, F., Manzano, I., Martín, D., Morín, J. y Roquero, E. 2012: “Contexto geomorfológico y principales rasgos tecnológicos de nuevos yacimientos del Pleistoceno Medio y Superior en el Valle Inferior del Manzanares (Madrid, España)". Estudios Geológicos 68 (1): 57-89.

Soler, N. y MAROTO, J. (1987): "Els nivells d'ocupació del Paleolític Superior a la cova de l'Arbreda (Serinyà, Girona)", Cypsela, 6: 221-228.

Tapias, F., Dorado, J. A., Gorosarri, J., Alarcón, A., López Recio, M., Morin, J. 2011 A: "Los depósitos cuaternarios asociados al valle del antiguo arroyo Abroñigal (cuenca del Manzanares, Madrid)". En M. Santonja (Cord.): Actas de las $5^{a}$ Jornadas de Patrimonio Arqueológico en la Comunidad de Madrid (Los primeros pobladores: Arqueología del Pleistoceno, Alcalá de Henares, 2008). M.A.R. Madrid: 255-267.

Tapias, F., Manzano, I., López Recio, M., Morín, J., Alarcón, A., Arteaga, C., Dapena, L., Del Moral, B., Garcia, M., Rolindes, A., Gorosarri, J., Dones, V., De Arcos, P. y Yravedra, J. 2011 B: "Puente de los tres ojos: aportaciones geoarqueológicas al estudio del cuaternario en el antiguo arroyo Abroñigal (cuenca del manzanares, Madrid)". En M. Santonja (Cord.): Actas de las $5^{a}$ Jornadas de Patrimonio Arqueológico en la
Comunidad de Madrid (Los primeros pobladores: Arqueología del Pleistoceno, Alcalá de Henares, 2008). M.A.R. Madrid 269-282.

UtRILLA, P. 1992: "Aragón/Litoral Mediterráneo. Relaciones durante el Paleolítico". Aragón/Litoral Mediterráneo. Intercambios culturales durante la prehistoria: 9-35.

Vega, L. G., Sevilla P., Colino F., De la Peña P., Rodriguez R., Gutiérrez F. y BÁrez S. 2011: "Nuevas investigaciones sobre los yacimientos Paleolíticos de la sierra norte de la comunidad de Madrid". En M. Santonja (Cord.): Actas de las $5^{a}$ Jornadas de Patrimonio Arqueológico en la Comunidad de Madrid (Los primeros pobladores: Arqueología del Pleistoceno, Alcalá de Henares, 2008). M.A.R. Madrid: 115-132.

VerneUIL, E. y LARTET, L. 1863: "Note sur un silex taillé trouve dans le diluvium des environs de Madrid". Bull. Soc. Geol. de France 2a XX: 698-702.

VILANOVA, J. 1891: "Objetos protohistóricos de Arganda del Rey". Boletín de la Real Academia de la Historia, 19: 513-516.

VILLAVERDE, V., 1979: "El Solutrense en el Pais Valenciano. Estado actual de su conocimiento". Tesis de Licenciatura, Universidad de Valencia: $369 \mathrm{p}$.

Wernert, P. y Pérez de Barradas, J. 1925: "El yacimiento paleolítico de San Isidro. Estudio bibliográfico-crítico". Revista de la Biblioteca Archivo y Museo del Ayuntamiento de Madrid 5: 31-68. 
